\title{
Controlling the Phase Segregation in Mixed Halide Perovskites through Nanocrystal Size
}

Andrés. F. Gualdrón-Reyes,,$_{, \dagger, \uparrow, \S}$ Seog Joon Yoon, ${ }^{\# \dagger}$ Eva M. Barea, ${ }^{\dagger}$ Said Agouram,"

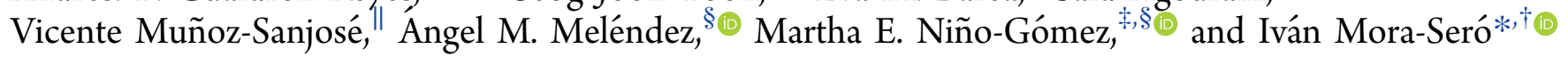

${ }^{\dagger}$ Institute of Advanced Materials (INAM), University Jaume I, Avenida de Vicent Sos Baynat, s/n, 12006 Castelló de la Plana, Castellón, Spain

${ }^{\ddagger}$ Centro de Investigaciones en Catálisis (CICAT), Universidad Industrial de Santander, Sede UIS Guatiguará, Piedecuesta, Santander C.P. 681011, Colombia

${ }^{\S}$ Centro de Investigación Científica y Tecnológica en Materiales y Nanociencias (CMN), Universidad Industrial de Santander, Piedecuesta, Santander C.P. 681011, Colombia

"Department of Applied Physics and Electromagnetism, University of Valencia, 46100 Valencia, Spain

\section{Supporting Information}

ABSTRACT: Mixed halide perovskites are one of the promising candidates in developing solar cells and light-emitting diodes (LEDs), among other applications, because of their tunable optical properties. Nonetheless, photoinduced phase segregation, by formation of segregated Br-rich and I-rich domains, limits the overall applicability. We tracked the phase segregation with increasing crystalline size of $\mathrm{CsPbBr}_{3-x} \mathrm{I}_{x}$ and their photoluminescence under continuous-wave laser irradiation $(405 \mathrm{~nm}, 10$ $\mathrm{mW} \mathrm{cm} \mathrm{cm}^{-2}$ ) and observed the occurrence of the phase segregation from the threshold size of $46 \pm 7 \mathrm{~nm}$. These results have an outstanding agreement with the diffusion length $(45.8 \mathrm{~nm})$ calculated also experimentally from the emission lifetime and segregation rates. Furthermore, through Kelvin probe force microscopy, we confirmed the correlation between the phase

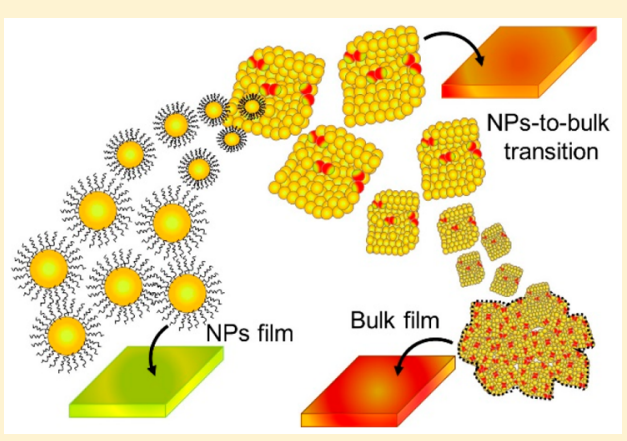
segregation and the reversible halide ion migration among grain centers and boundaries. These results open a way to achieve segregation-free mixed halide perovskites and improve their performances in optoelectronic devices.

$\mathrm{T}$ he study of halide perovskite devices has been a hot topic during recent years, as indicated by the fast increase of the photoconversion efficiency in perovskite solar cells (PSCs) to values close to $23 \% .{ }^{1}$ Halide perovskites demonstrate a benign defect physics inducing low nonradiative recombination and notable optical properties, such as high absorption cross section, charge carrier diffusion length, low exciton dissociation energy, and so on. ${ }^{2}$ These properties make halide perovskites promising materials not just for photovoltaic applications but also for other optoelectronic devices with enhanced properties, such as light-emitting diodes (LEDs), ${ }^{3,4}$ optical amplifiers, ${ }^{5,6}$ and lasers. ${ }^{7,8}$ One of the most interesting properties of the perovskite family of materials is their versatility. By the combination of cations ( $A$ and $B$ ) and halide anions $(\mathrm{H})$ of the perovskite general formula $\mathrm{ABH}_{3}$, a broad range of materials with different properties and band gaps can be attained. ${ }^{9}$ Thus, the band gap can be continuously tuned just by adjusting $x$ in the general formula $\mathrm{ABH}_{3-x} \mathrm{H}^{\prime}{ }_{x}$ where $\mathrm{H}$ and $\mathrm{H}^{\prime}$ are two different halides, commonly I and $\mathrm{Br}$ or $\mathrm{Br}$ and $\mathrm{Cl}^{10,11}$

However, under photoirradiation or with an applied bias, mixed halide perovskites present a main limitation due to the segregation of the $\mathrm{H}_{3-x} \mathrm{H}^{\prime}{ }_{x}$ phase into two phases, namely, $\mathrm{H}$ rich and $\mathrm{H}^{\prime}$-rich phases, as was first reported by Hoke et al. ${ }^{12}$ In the case of $\mathrm{Br}-\mathrm{I}$ mixed halide perovskites, the phase segregation involves halide segregation to produce lower-band gap I-rich domains and higher-band gap Br-rich domains. ${ }^{12-15}$ Previous works have demonstrated that the two reversible competitive process, phase segregation (in tens of second) and its recovery (in tens of minutes), can be tracked through spectroscopic tools such as absorption, transient absorption, and photoluminescence (PL). ${ }^{14,16}$ Halide segregation occurs through halide migration, and the segregated domains locate at the grain boundaries to release lattice strain. ${ }^{14,17}$ Furthermore, I-rich segregated domains act as recombination centers for photogenerated charge carriers irrespective of the carrier generation sites into other mixed or Br-rich domains. ${ }^{16,18}$ The later issue has been reported to impact negatively on the performance of mixed halide perovskite-based solar cells, which leads to trapping of the carriers and hinders the electron

Received: November 14, 2018

Accepted: November 27, 2018

Published: November 27, 2018 
extraction in devices. ${ }^{19}$ Hence, photovoltaic parameters such as open-circuit voltage and photoconversion efficiency are seriously affected. ${ }^{18,19}$ This fact limits the optimization of the perovskite band gap facing the development of tandem devices $^{15,20}$ that require the high band gaps attainable by the mixed halide perovskites ${ }^{21}$ and the development of a wide range of colors, potentially reachable by perovskite LEDs, for applications in on-demand illumination or in white LEDS with different color temperature, to cite just a couple of examples that require an optimum control of the emitted light color.

To minimize the photoinduced halide segregation effect in mixed halide perovskites and to increase their applicability in optoelectronics, some possibilities have been investigated. ${ }^{22}$ Alloying cations into the perovskite matrix (e.g., such as methylammonium (MA), cesium, and/or formamidinium (FA)) reduces or minimizes the time-dependent absorption/ emission change under photoirradiation by increasing entropic contributions. $^{23-25}$ In addition, various efforts were made to stop or minimize the phase segregation as follows: (i) increasing the crystalline size and the microstrain by combining $\mathrm{Pb}^{2+}$ with $\mathrm{Sn}^{2+}$ cations into perovskite matrix; ${ }^{26}$ (ii) growing the perovskite grains on nonwetting polybis(4phenyl)(2,4,6-trimethylphenyl)amine (PTAA) to improve the crystallinity and grain size to retard the segregation; ${ }^{27}$ and (iii) inducing surface passivation of perovskites with $\mathrm{K}^{+}$cations to minimize the site at grain boundaries where the phase segregation occurs. ${ }^{28}$

Despite these interesting options, a further understanding of the phase segregation process providing clear strategies in obtaining phase segregation-free perovskite films in all mixed halide compositions is still necessary. Recent theoretical results highlight the interplay between induced phase segregation and crystalline size. ${ }^{17,29}$ Bischak et al. demonstrated that large electron-phonon coupling induces phase segregation by releasing the lattice strain and that the generated polarons move to segregated domain with larger mobility, so the segregated domains are stabilized before the domains recover to the initial mixed perovskite state. ${ }^{17}$ Draguta et al. reported a correlation between the carrier diffusion length $(L)$ and the rate of segregation $(K)$ as ${ }^{29}$

$$
K=K_{\text {sat }}\left[1-\exp \left(-4 / 3 \pi L^{3} \rho\right)\right]
$$

where $K_{\text {sat }}$ and $\rho$ are the saturated segregation rate and the photogenerated carrier density, respectively. Equation 1 implies that lowering the carrier diffusion length will tend to decrease the rate of segregation, and thus the segregation rate could be similar to the recovery rate for being at the initial mixed halide perovskite state. ${ }^{29}$ Draguta et al. ${ }^{29}$ developed through density functional theory (DFT) calculation a diffusion length-dependent phase diagram differentiating between the segregation region and segregation-free region. This implies that there is a segregation-free region for mixed halide perovskites constituted by nanoparticles of certain crystalline size below a threshold. They also experimentally supported the segregation-free region by showing for the first time stable emission from $\mathrm{CsPbBr} \mathrm{Pb}_{1.5} \mathrm{I}_{1.5} \mathrm{NPs}$. However, the occurrence of phase segregation dependent on crystalline size at a certain threshold has not been experimentally determined.

To find the threshold size for segregation-free perovskites, and consequently develop devices with real band gap tunability, we have studied, by employing different characterization techniques, the size-dependent phase segregation process, and we have found the borderline to induce the phase segregation. To perform the experiment, we synthesized nanocrystalline-sized $\mathrm{CsPbX}_{3}$ nanoparticles (NPs), following the procedure developed by Kovalenko and co-workers, ${ }^{30,31}$ and formed bulk films through annealing the $\mathrm{CsPbX}_{3}$ deposited NP thin films (termed nanoparticle film). ${ }^{32}$ Inorganic $\mathrm{CsPbX}_{3} \mathrm{NPs}$ exhibit higher stability than the previously developed hybrid organic-inorganic perovskite NPs. Kovalenko and co-workers demonstrated that by using $\mathrm{CsPbX}_{3} \mathrm{NPs}$ it is possible to tune their band gaps by varying the halide composition in order to control the valence band maximum. ${ }^{30,31}$ Through halide composition variation, the band gap has been modulated in the entire visible region. Hoffman et al. reported that, after an annealing process, $\mathrm{CsPbBr}$ NPs make bulk crystalline domains larger than $100 \mathrm{~nm}$ through agglomeration and by absorbing smaller NPs in a process analogous to Ostwald ripening. ${ }^{32}$ In this Letter, by linking the morphology, crystallinity, and photophysical properties of $\mathrm{CsPbBr}{ }_{3-x} \mathrm{I}_{x}$, we have demonstrated the gradual transition from the range of crystalline sizes that, after irradiation, is free from phase segregation to values of the crystalline size in which, after irradiation, a phase segregation is generated. These results open new possibilities to control the photoinduced phase segregation process of $\mathrm{CsPbBr}_{3-x} \mathrm{I}_{x}$ perovskite films for enhanced stability and performance in optoelectronic devices.

Morphological and Photophysical Properties of $\mathrm{CsPbBr}_{3-x} I_{x}$ Nanoparticles. Hot-injection synthesis of perovskite $\mathrm{NPs}^{30,31}$ has been used to prepare stable colloidal high-luminescent materials. For our study, we synthesized five different type of colloidal $\mathrm{CsPbBr}_{3-x} \mathrm{I}_{x}$ NPs in hexane, varying the $\mathrm{Br} / \mathrm{Br}+\mathrm{I}$ ratio in the semiconductor as seen in Figure S1. The color change from green (high Br-content) to red (high I-content) in the nanoparticles shows clearly that their optical properties can be tuned by halide modification. ${ }^{30}$ Figure S2 displays TEM measurements for five different types of $\mathrm{CsPbBr}_{3-x} \mathrm{I}_{x}$ perovskite nanoparticles $(x=3,2.67,1.44,0.63$, and 0$)$. In all cases, nanometer-sized $(11.8-14.3 \mathrm{~nm})$ cubic nanoparticle cores were obtained. In Table S1, the chemical composition of perovskite NPs (in atom \%) was estimated from EDX analysis. $\mathrm{The} \mathrm{Br} /(\mathrm{Br}+\mathrm{I})$ ratio was decreased by increasing the amount of iodine without any modification of $\mathrm{Pb}$ and $\mathrm{Cs}$ nominal content, just to maintain a stoichiometric $\mathrm{CsPbX}_{3}$-type structure. The nominal halide composition, based on the ratio of $\mathrm{I}$ and $\mathrm{Br}$ precursors used in the synthesis, is in excellent agreement with those achieved from EDX measurements, corroborating the incorporation of the halide content into the perovskite samples. The composition was double checked considering the XRD pattern (see Table S1). ${ }^{33}$

To obtain information about the quality and the halide composition-dependent photophysical features of the $\mathrm{CsPbBr}_{3-x} \mathrm{I}_{x}$ NPs, a spectroscopic tracking was performed. Figure S3A shows the evolution of the optical absorption for the perovskite nanoparticles, where a red-shift in the absorption offset was achieved by increasing the iodine content. This trend was also observed for the emission feature for each composition (Figure S3B), which was associated with the band gap narrowing into the semiconductor. Furthermore, the emission spectra depict a symmetric peak for each $\mathrm{CsPbBr}_{3-x} \mathrm{I}_{x}$ composition with a $30 \pm 5 \mathrm{~nm}$ full width at half-maximum (FWHM), being a main characteristic of monodisperse solid solutions. ${ }^{31}$ The band edge and emission peak position were estimated from absorption and emission measurements, respectively; they are summarized in Table S2. As can be seen, only small differences between these values 
A

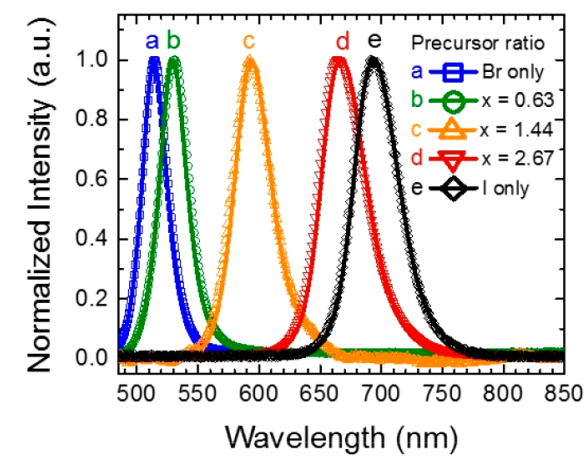

C

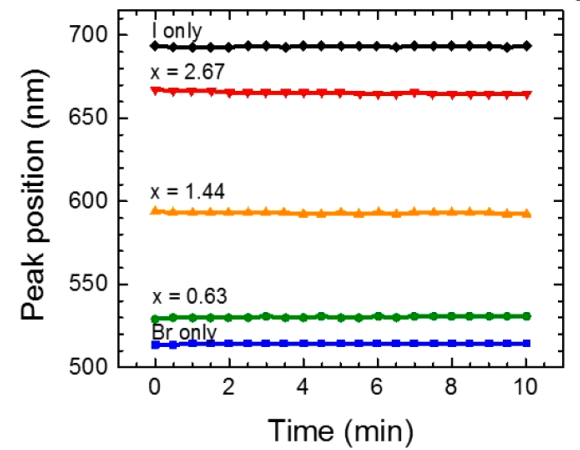

B

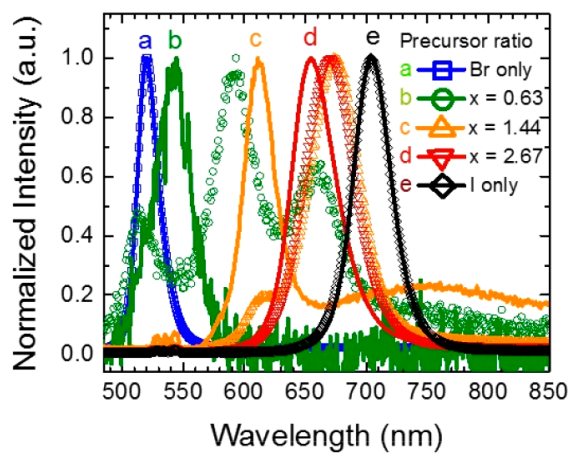

D

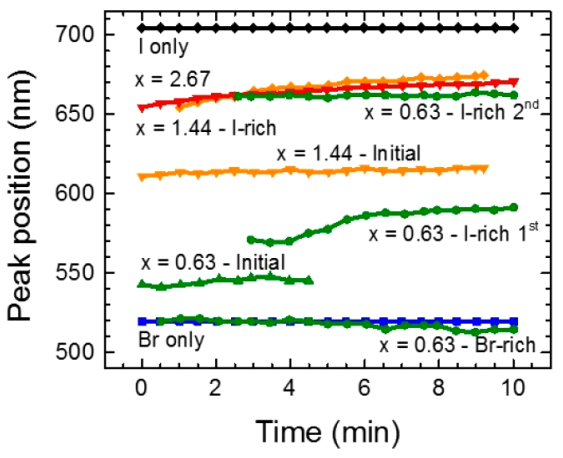

Figure 1. (A and B) Normalized photoluminescence spectra obtained under continuous wave (CW) excitation $\left(405 \mathrm{~nm}, I_{\text {exc }}=10 \mathrm{~mW} \mathrm{~cm}{ }^{-2}\right)$ with initial spectra (line) and spectra after $10 \mathrm{~min}$ of irradiation (symbols) and (C and D) peak position as a function of time for $\mathrm{CsPbBr}_{3-x} \mathrm{I}_{x}$ (A and C) NPs and (B and D) bulk films after annealing at $225{ }^{\circ} \mathrm{C}$ for 180 s. Note that the emissions from annealed bulk CsPbI ${ }_{3}$ or $\mathrm{CsPbBr}_{3-x} \mathrm{I}_{x}$ films $(x=2.67)$ are from minor cubic phase with dark red color. After formation of the bulk film, the major crystalline phase of $\mathrm{CsPbI}_{3}$ or $\mathrm{CsPbBr}_{3-x} \mathrm{I}_{x}$ films $(x=2.67)$ changes to orthorhombic phase with yellow color.

were evidenced, achieving a high agreement in the optical absorption and emission features of the samples. On the other hand, after calculation of the band gap from the emission peak position for each composition of perovskite, the band gap narrowing was clearly evidenced as a function of the halide composition (Figure S3C). Thus, the $\mathrm{CsPbBr}_{3-x} \mathrm{I}_{x}$ NPs cover the visible spectral region of $1.8-2.4 \mathrm{eV}$, commonly reported for mixed halide perovskites. ${ }^{12,15}$ Lastly, after measurement of the photoluminescence emission quantum yield (PLQY) of the samples (Figure S3D), a range between 20 and $85 \%$ was reached, indicating highly emissive perovskite nanocrystals (see Table S2).

Morphological, Crystallinity, and Photophysical Features of $\mathrm{CsPbBr}_{3-x} I_{x}$ Nanoparticles and Bulk Films. As described above, a main issue in mixed halide perovskites is the presence of photoinduced phase segregation negatively affecting the photovoltaic parameters in optoelectronic devices. ${ }^{18}$ The segregation effect has appeared in methylammonium, ${ }^{12,14,16}$ formamidinium, ${ }^{12}$ and cesium mixed halide perovskite films, ${ }^{13,29}$ forming iodine-rich and bromine-rich domains, which have been spectroscopically tracked. Nonetheless, the current efforts have focused on the study of film properties to investigate the phase segregation (morphological and chemical composition points of view). ${ }^{34,35}$

The preparation of $\mathrm{CsPbBr}_{3-x} \mathrm{I}_{x} \mathrm{NP}$ films and their transformation to bulk films under annealing process can give valuable evidence about the threshold size for the occurrence of the photoinduced phase segregation. As a first step in this study, NP films were prepared by drop-casting five different $\mathrm{CsPbBr}_{3-x} \mathrm{I}_{x}$ NP solutions on glass slides. Then, bulk films were achieved by annealing the NP films at $225^{\circ} \mathrm{C}$ for 180 s. Similar to NP solutions (Figure S1), a red-shift of the PL feature is presented (Figure S4) as a function of the iodine content in perovskites. However, PL is significantly reduced after annealing because of the NP agglomeration and formation of bulk film with lower PLQY. We can conclude that nonradiative recombination centers are created, reducing the PL intensity in the bulk films. Lower values of PLQY in bulk films $(<1 \%)$ were also observed elsewhere. ${ }^{36}$ Furthermore, it is worth noting that the cubic phase of iodine-rich perovskites $(x=2.67$ and 3) NPs with dark red color was preserved on nanoparticle films. In contrast, after annealing, yellow colored bulk film were observed because of the change of the crystalline phase from cubic to orthorhombic phase in $\mathrm{CsPbI}$-based perovskites, which is thermodynamically preferred in the bulk at room temperature. ${ }^{37,38}$

The variation of halide composition and effects of NP-tobulk film transition were observed to influence the photophysical properties of the $\mathrm{CsPbBr}_{3-x} \mathrm{I}_{x}$ perovskites. Here, the normalized emission features of the $\mathrm{Cs} \mathrm{PbBr}_{3-x} \mathrm{I}_{x}$ NPs and bulk films were monitored before and after $10 \mathrm{~min}$ of photoirradiation and plotted in Figure 1 (whole time-dependent non-normalized emission spectra are in Figure S5). Before photoirradiation, the emission peak position for all perovskite nanoparticles was red-shifted as a function of the iodine content (Figure 1A). After 10 min under photoirradiation, the peak position was stable (Figure $1 \mathrm{C}$ ) with a decrease in the PL intensity and a slight blue-shift of around $4 \mathrm{~nm}$ (see Figure S5). This effect could be caused by some exposure of the nanoparticle surface to the environment, inducing some 
perovskite oxidation. ${ }^{29}$ The latter was confirmed through the emission features of $\mathrm{CsPbBr}_{3-x} \mathrm{I}_{x}$ embedded PMMA films (Figure S6), deducing that the ligand-exchange process for depositing NP layers does not affect the NP stability. The stable PL from $\mathrm{Cs} \mathrm{PbBr}_{1.5} \mathrm{I}_{1.5}$ NPs against phase segregation was reported earlier. ${ }^{29}$ We checked that the stable PL against phase segregation can be obtained with various $\mathrm{Br} / \mathrm{I}$ halide compositions. Therefore, nanometer-sized (11-14.3 nm) $\mathrm{CsPbX}_{3} \mathrm{NPs}$ with various $\mathrm{Br} / \mathrm{I}+\mathrm{Br}$ composition maintained the PL peak position, which means that NP films are free from the phase segregation of mixed halide perovskites.

Conversely, under the same irradiation time, the initial emission peak for $\mathrm{CsPbBr}_{3-x} \mathrm{I}_{x}$ bulk films was bleached, and a continuous red-shift with an increase of PL intensity was achieved (Figures 1B and S5D,F,H). Note that the red shift of initial emissions from nanoparticle to bulk film is due to formation of conduction/valence bands through agglomeration of NPs, so that the decreased band gap reflected red-shifted emission. According to previous work, the evolution of PL toward longer wavelengths (Figure 1D) indicates the presence of phase segregation in the mixed halide preovskite domains, forming segregated Br-rich and I-rich domains and then transferring free carriers to the I-rich domains. ${ }^{12,14,16}$ Similar irradiation time-dependent emission change was observed in conventional one-step solution processes to form a bulk $\mathrm{CsPbX}_{3}$ film from precursor solution through annealing. ${ }^{15}$ When the Br-rich and I-rich domains are produced, the rise of two emission peaks at low- and high-energy regions is expected. ${ }^{12,15-17}$ To track the occurrence of phase segregation, absorption measurements were also carried out after irradiation at $10 \mathrm{~mW} \mathrm{~cm}{ }^{-2}$ for $10 \mathrm{~min}$. As can be seen, the absorption peak of $\mathrm{CsPbBr}_{3-x} \mathrm{I}_{x}$ nanoparticle films rested unchanged after recovering in dark conditions (Figure S7A,C). There was no change of absorption features with respect to the absorption in nonirradiated perovskite nanoparticles (Figure S7C). In contrast, in the case of the bulk films (Figure S7B,D), an increment of the absorption at lower and higher wavelengths appeared, corresponding to the Br-rich and I-rich phases. ${ }^{16}$ The halide segregated feature of the mixed halide perovskites was more evident after measuring the dark recovery after 45 min (Figure S7D). According to Kamat and co-workers, ${ }^{16}$ the absence of the Br-rich phase in the emission feature can be attributed to a rapid transport and recombination of photogenerated charge carriers into the I-rich phase, which is energetically favorable to appear under phase segregation.

Interestingly, three different emission peaks were formed for the $\mathrm{CsPbBr}_{3-x} \mathrm{I}_{x}$ bulk film $(x=0.63)$ around 512, 593, and 662 $\mathrm{nm}$, after the initial peak around $544 \mathrm{~nm}$ was bleached (see Figure $1 \mathrm{~B}$ ). The peak at the high-energy region is ascribed to the Br-rich phase, while a doublet at low-energy region corresponds to the I-rich phase. By considering that (i) the NP agglomeration is not a uniform process on the film surface after annealing and (ii) the iodide migration to positive grain boundaries formed during the agglomeration has been reported to occur under phase segregation, ${ }^{13,34}$ we suggest that at least two different kinds of I-rich phases (different amount of iodide in the segregated domains) were created during the NP-to-bulk transformation.

To observe more in depth the effect of the halide composition and annealing process on the crystallinity of the as-prepared samples, XRD analyses were also performed. Figure $\mathrm{S} 8$ shows the XRD patterns of the $\mathrm{CsPbBr}_{3-x} \mathrm{I}_{x} \mathrm{NP}$ films deposited on glass with (Figure S8B) or without (Figure S8A) the annealing process. For all materials, two main peaks were achieved at low and high Bragg angles, associated with the characteristic 100 and 200 planes of the perovskite lattice, respectively. ${ }^{39}$ These planes indicate the presence of the cubic phase in the NP film, which is expected at synthesis temperatures between 100 and $200{ }^{\circ} \mathrm{C} .^{30,40}$ Furthermore, a red-shift in the Bragg angle was evidenced as a function of the $\mathrm{Br}$ content into $\mathrm{CsPbBr}_{3-x} \mathrm{I}_{x}$ perovskites (Figure S8). This is ascribed to the lattice contraction of the semiconductor by the presence of the smaller ionic radius of bromide (in comparison with iodide)..$^{31}$ Under the annealing process at $225^{\circ} \mathrm{C}$ (Figure $\mathrm{S} 8 \mathrm{C})$, the XRD peaks for $\mathrm{Br}$-rich $\mathrm{CsPbBr}{ }_{3-x} \mathrm{I}_{x}$ perovskites $(x=$ $0,0.63$, and 1.44) became thinner than the initial NPs (Figure $\mathrm{S} 8 \mathrm{D}$ ), which is an indication of the NP agglomeration and grain growth. Five new peaks appeared in the XRD patterns of the high I-rich samples ( $x=2.67$ and 3), and a decrease in the intensity of the cubic phase signals was achieved, attributed to the $\alpha$-perovskite phase transformation into the $\delta$-orthorhombic phase. $^{37}$ This is supported by the color change from dark brown to light yellow described above for the I-rich perovskites (see Figure S4). Concerning the NP size growth under annealing, SEM images of the four types of $\mathrm{CsPbBr}_{3-x} \mathrm{I}_{x}$ perovskites $(x=0,0.63,1.44$, and 2.67$)$ display big crystals with average grain size between 100 and $200 \mathrm{~nm}$ (Figure S9A$\mathrm{H})$. For the case of $\mathrm{CsPbI}_{3}$ perovskite, microwires with an average size of around $144 \mathrm{~nm}$ were obtained (Figure S9I-L). This clearly agrees with the XRD patterns of annealed films, which corroborates the NP agglomeration to produce larger crystalline grains (see Figure S11).

Effect of Size on the Phase Segregation of $\mathrm{Cs} P b B r_{3-x} I_{x}$ Films. Considering the fact that the annealing process induces phase segregation in mixed halide perovskites during their NP-tobulk transformation (in accord with emission measurements explained above), PL profiles of $\mathrm{CsPbBr}_{3-x} \mathrm{I}_{x}$ nanoparticle films $(x=0.63)$ with different sizes were tracked by varying the annealing time $(0,5,10,30,60$, and $180 \mathrm{~s})$. As is shown in Figure $2 \mathrm{~A}$, increasing the annealing time "turns off" the PL of the films, which can be associated with the agglomeration of NPs. By using TEM (Figure S2C,D) and SEM images (Figure $\mathrm{S} 12 \mathrm{~A}-\mathrm{N})$, we determined that the crystalline size was $11.8 \pm$ $1.1 \mathrm{~nm}$ for $\mathrm{CsPbBr}_{3-x} \mathrm{I}_{x} \mathrm{NP}$ films (Figure 2B), generating bigger grains of $301 \pm 126 \mathrm{~nm}$ size after $180 \mathrm{~s}$ of annealing (Figure 2C). In this sense, we were able to observe the sequential change in the crystallinity of the $\mathrm{CsPbBr}_{3-x} \mathrm{I}_{x}$ films as a direct response of the NP-to-bulk transition. Also, Figure $2 \mathrm{D}$ displays the evolution of the grain size as a function of the annealing time. This demonstrates that big grains are formed in the bulk films through NP agglomeration (Figure S12). The large grains are generated with the increase of annealing time through material migration from smaller to larger NPs, which is more energetically favorable in the presence of larger particles than those smaller ones, as reported earlier. ${ }^{32}$

In Figure 2E, we present additional IR measurement results for every film preparation stage in order to track the presence of the QDs organic capping ligands. Independent of the preparation stage, peaks from the capping ligands were found, i.e., peaks at 2920, 2850,1641, and $1465 \mathrm{~cm}^{-1}$ represent $-\mathrm{CH}_{2}$ asymmetric stretch, $-\mathrm{CH}_{2}$ symmetric stretch, $\mathrm{C}=\mathrm{C}$, and $-\mathrm{CH}_{3}$ stretch, respectively. The signal from the ligands presents the highest decrease after the ligand exchange process and a low decrease with the annealing time (Figure 2E), indicating the presence of ligands even after annealing. Hoffman et al. also 


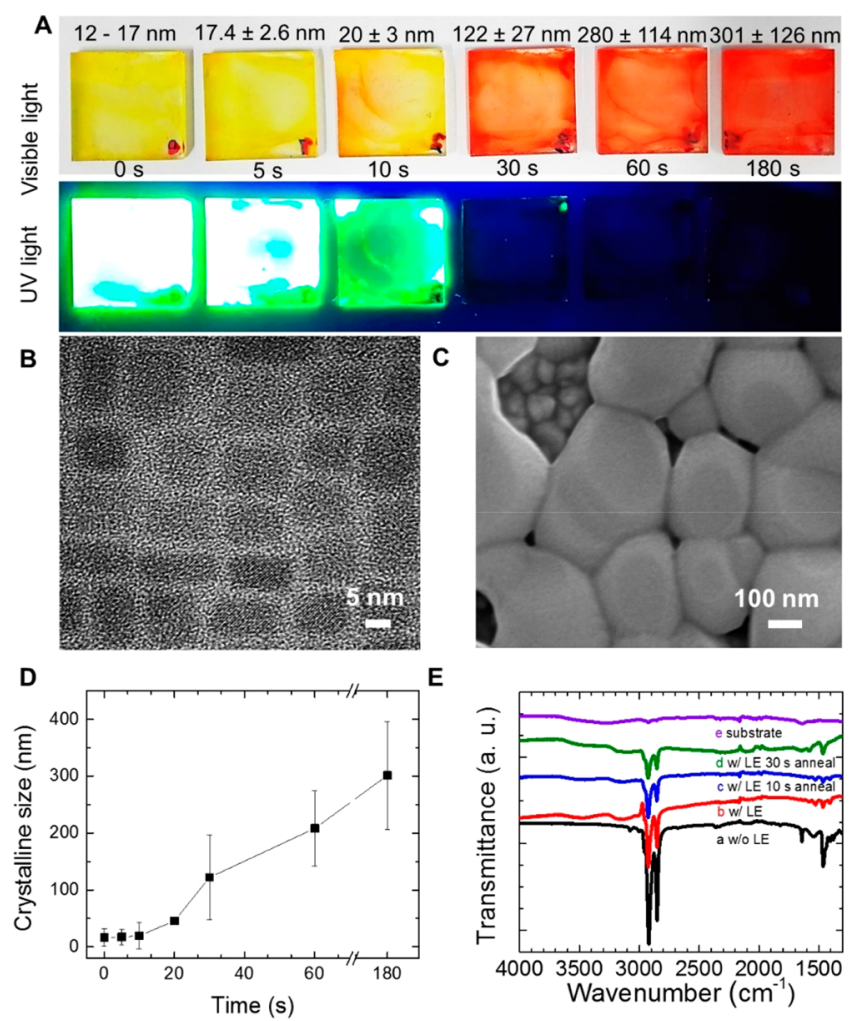

Figure 2. (A) Photographs of $\mathrm{CsPbBr}_{3-x} \mathrm{I}_{x}$ films $(x=0.63)$ with different crystalline sizes, taken under visible light and UV light. (B) TEM image of $\mathrm{CsPbBr}_{3-x} \mathrm{I}_{x}$ NPs $(x=0.63)$. (C) SEM image of $\mathrm{CsPbBr}_{3-x} \mathrm{I}_{x}$ bulk film $(x=0.63)$ through agglomeration of nanoparticles upon annealing. (D) Crystalline size determined by SEM of the $\mathrm{CsPbBr}_{3-x} \mathrm{I}_{x}$ bulk films obtained at $225{ }^{\circ} \mathrm{C}$ during different annealing times. (E) FTIR spectra of the $\mathrm{CsPbBr}_{3-x} \mathbf{I}_{x}$ film $(x=0.63)$ (a) without ligand exchange (LE), with subsequent (b) ligand exchange, and (c and $d$ ) annealing process as a function of time to form bulk film.

reported the presence of a ligand on grains after formation of bulk film through the annealing process. ${ }^{32}$

Figure S10 shows the XRD patterns of the perovskite NP films annealed at $225{ }^{\circ} \mathrm{C}$ with varying grain size. Here, the (100) and (200) planes were achieved for all the samples, establishing the cubic phase as the crystalline structure. ${ }^{39}$ As also shown in Figure S11A, the decrease of the FWHM of the peaks corresponding to the (100) plane indicates an increasing crystalline size as a function of the annealing process. It also supports the conclusions that the NP agglomeration forming the bulk film occurred during the annealing process.

On the other hand, to understand the correlation between nanoparticle size and the phase segregation, in Figure 3 we present the photophysical features of $\mathrm{CsPbBr}_{3-x} \mathrm{I}_{x}$ films $(x=$ 0.63 ) as a function of the crystalline size, as already shown in Figure 2. The emission spectra of the as-prepared $\mathrm{CsPbBr}_{3-x} \mathrm{I}_{x}$ bulk films before and after photoirradiation are shown. Details of the time-dependent non-normalized emission spectra with the different sizes are presented in Figure S13. As shown in Figure $3 \mathrm{~A}$, the segregation effect was not present in the $\mathrm{CsPbBr}_{3-x} \mathrm{I}_{x} \mathrm{NP}$ films with the crystalline sizes up to $120 \pm 3$ $\mathrm{nm}$. However, as shown in Figure 3B, after $20 \mathrm{~min}$ of irradiation, slow occurrence of the phase segregation emerged when the size of the $\mathrm{CsPbBr}_{3-x} \mathrm{I}_{x}$ films reached $46 \mathrm{~nm}$. Through pseudo-first-order kinetic analysis by monitoring emission evolution from the segregated I-rich domain, we were able to obtain a constant rate of $K=1.53 \times 10^{-3} \mathrm{~s}^{-1}$ in the case of $46 \pm 7 \mathrm{~nm}$ grain size (Figure S15B). In contrast, in the case of a larger size $(301 \pm 126 \mathrm{~nm})$, the constant rate was $K_{\text {sat }}=$ $4.54 \times 10^{-3} \mathrm{~s}^{-1}$ (Figure S15C). In Figure 3C, we can see a different trend with respect to Figure 3A. The emission from larger grains $(122 \pm 27 \mathrm{~nm}$ or larger) changes with photoirradiation. Note that the agglomeration process was not homogeneous in the entire bulk film surface, and therefore, we suggest that at least two different grain boundary contents could be formed in the bulk crystals to produce two diverse Irich phases. In Figure S14, absorption spectra after photoirradiation also support the occurrence of phase segregation from the perovskite with the crystalline size greater than $46 \pm$ $7 \mathrm{~nm}$ by observing the recovery of optical properties. Furthermore, if the grain size would be greater than $122 \mathrm{~nm}$, the emission evolution from the segregated I-rich domain seems to be similar (Figure S13D-F). Similar emission evolutions shown in Figure S13D-F demonstrate that there is a saturation of segregation rate. We can suggest that if the grain size would be equal or larger than $46 \pm 7 \mathrm{~nm}$, the grain size may exceed the diffusion length. This means that the electron/hole mobility would not be confined in the grain and the charge would diffuse with its own diffusion length. The electron/hole mobility could be confined into the grain around $46 \pm 7 \mathrm{~nm}$, the grain size being similar to the charge diffusion length. To support the comparability between the grain size and charge diffusion length $(L)$, we calculated the diffusion length by following eq 1 and obtained the diffusion length as $45.8 \mathrm{~nm}$ (see details in Supplementary Note in the Supporting Information). Thus, the observed grain size for transition
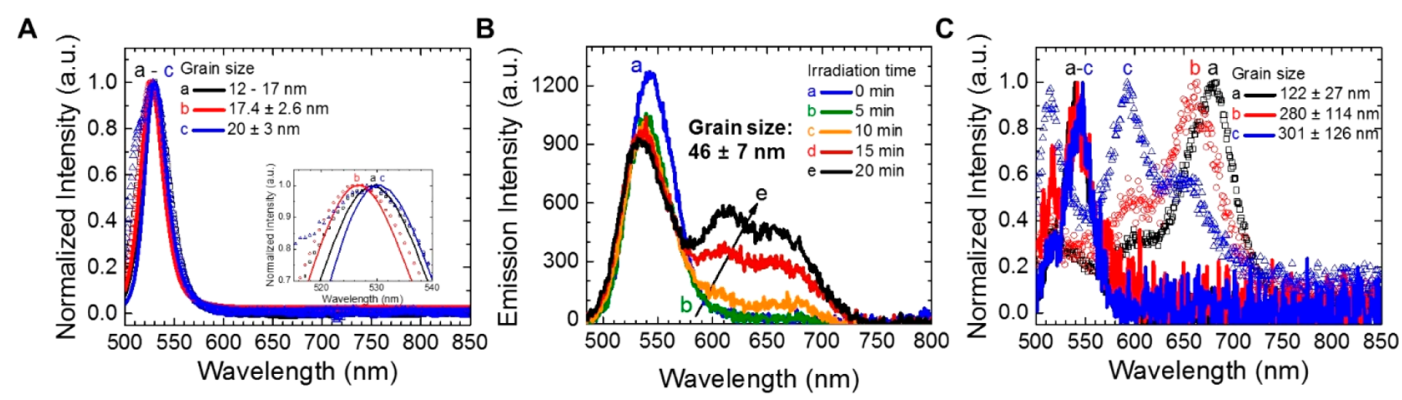

Figure 3. Normalized emission spectra of the $\mathrm{CsPbBr}_{3-x} \mathrm{I}_{x}$ bulk films $(x=0.63)$, from different sizes with continuous wave (CW) laser irradiation $\left.\left(I_{\text {exc }}=10 \mathrm{~mW} \mathrm{~cm}\right)^{-2}\right)$. (A and C) Initial emission (line) and emission after 10 min of irradiation (dots) were assigned. (A) Emission spectra from films with grain sizes up to $19.5 \mathrm{~nm}$. (B) Tracking of emission feature of the $\mathrm{CsPbBr}_{3-x} \mathrm{I}_{x}$ perovskite film with $46 \pm 7$ nm grain size, during 20 min of CW excitation. (C) Emission spectra from films with grain sizes greater than $100 \mathrm{~nm}$. 

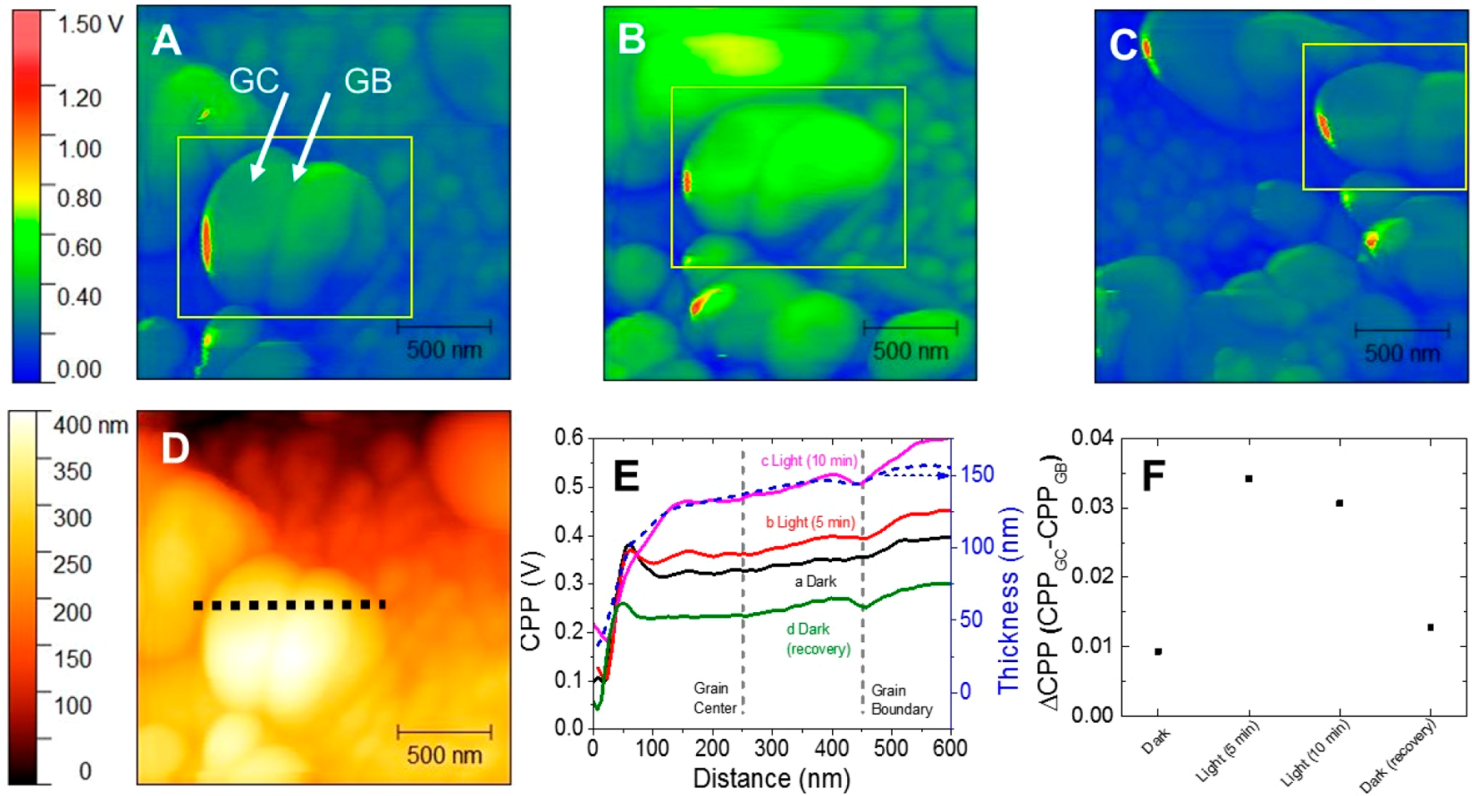

Figure 4. Kevin probe force microscopy (KPFM) images obtained for $\mathrm{CsPbBr}_{3-x} \mathrm{I}_{x}$ bulk films $(x=0.63)$ with $122 \pm 27 \mathrm{~nm}$ grain size, under (A) dark conditions, (B) $10 \mathrm{~min}$ of photoirradiation, and (C) after recovery conditions in the dark. (D) Topographic image and (E) crosssectional profile of contact point potential (CPP) through the dotted line in panel D for the bulk film. (F) CPP difference ( $\triangle$ CPP) between the grain center (GC) and grain boundary (GB) in the bulk film during the dark/illumination/recovery processes. The positions of grain boundary and grain center were marked in panel $\mathrm{E}$ as dashed lines.

between the segregation-free zone and phase segregation behavior has an outstanding agreement with the diffusion length calculated from eq 1 and experimentally determined average lifetime for PL, $K$, and $K_{\text {sat }}{ }^{29}$

On the other hand, note that the phase segregation happens when the grain size reached the size $46 \pm 7 \mathrm{~nm}$, and the capping ligands play a minor role. As shown in Figure 2E, IR spectra at each preparation stage, we found the gradual decrement of the ligand, but still the ligand was present after the bulk film preparation. We could observe the presence of the ligand in any case with and without the phase segregation, but segregation is induced only after forming bulk films, when the grain size becomes $46 \pm 7 \mathrm{~nm}$ or larger, highlighting the major role of crystal size in this process. This minor role of the ligand in comparison with the crystal size has been also verified by carrying out this analysis in samples without ligand exchange, observing phase segregation after annealing despite the higher amount of capping ligands (see Figure S16).

KPFM Characterization. To gain a deeper understanding about the assumption of the halide ion segregation in the bulk film, after-annealing Kevin probe force microscopy (KPFM) images (Figure 4) were achieved for the $\mathrm{CsPbBr}_{3-x} \mathrm{I}_{x}$ bulk films $(x=0.63)$ annealed with $120 \pm 27 \mathrm{~nm}$ grain size, in the absence and presence of photoirradiation. Under dark conditions (Figure 4A), the contact point potential (CPP) was homogeneous on the bulk film, either at grain boundary (GB) or grain center (GC). Herein, we will use the term GB as an intergrain grain boundary, where different grains meet each other, and GC is the position in the grain far from the GB. Under $5 \mathrm{~min}$ (Figure S17A) and $10 \mathrm{~min}$ of photoirradiation (Figure 4B), the CPP at the GC was increased in comparison with the CPP at GB (Figures $4 \mathrm{~F}$ and S17B). After the photoirradiation was turned off, the CPP of the bulk film was relaxed back (Figure 4C). Under photoirradiation, differences of the CPP between the GC and the GB were associated with charge compensation due to the anion movement. To date, it has been reported that photoirradiation induces ion migration. ${ }^{41}$ Among the ions, mostly halide ions as bromide (0.17$0.25 \mathrm{eV})$ and iodide $(0.23-0.43 \mathrm{eV})$ have lower activation energy to promote migration as compared to other ions into the perovskite. ${ }^{33}$ A cross-sectional profile was achieved from the topography image of $\mathrm{CsPbBr}_{3-x} \mathrm{I}_{x}$ grains, where the contact point potential was monitored as a function of the position labeled by a dashed line on the film surface (Figure 4D,E). At about $450 \mathrm{~nm}$, a potential drop was achieved, typical from the $\mathrm{CPP}$ at $\mathrm{GB} .{ }^{34}$ At $250 \mathrm{~nm}$ of labeled distance (CPP at GC), compared to CCP at GB, a progressive increase of the potential was observed under photoirradiation. This trend can be better evidenced from the potential difference for the bulk film under dark/photoirradiation/recovery conditions. As shown in Figures $4 \mathrm{~F}$ and $\mathrm{S} 17 \mathrm{~B}$, the CPP at GB did not change as significantly as did CPP at GC, considering that the overall potential change occurred in the GC. By subtracting the $\mathrm{CPP}$ of $\mathrm{GC}$ and $\mathrm{GB}$ (denominated as $\mathrm{CPP}_{\mathrm{GC}}$ and $\mathrm{CPP}_{\mathrm{GB}}$, respectively), GC exhibited the highest $\mathrm{CPP}$ under photoirradiation. Considering the local observation of bulk crystals performed by other authors, a higher CPP in GC is an indication of a halide deficiency in this region due to the halide migration to the GB. This means that GB is a halide-enriched region, which triggers the phase segregation, while the halidedeficient GC retards the phase segregation. ${ }^{14,34}$ In the case of $\mathrm{CsPbBr}_{3}$ bulk film (see Figure $\mathrm{S} 18$ ), $\mathrm{Br}$ migration is also expected under illumination conditions. In fact, a change, $\triangle \mathrm{CPP}$, between GC and GB is detected when comparing the dark and light conditions (see Figure S19), indicating this illumination triggered migration effect. However, for $\mathrm{Cs} \mathrm{PbBr}_{3}$, $\Delta \mathrm{CPP}$ decreases when samples under dark conditions are illuminated (see Figure S19), opposite what is observed in mixed halide samples (see Figure 4F). It has been reported that anion photosegregation is mediated by vacancies, ${ }^{42}$ as ion 

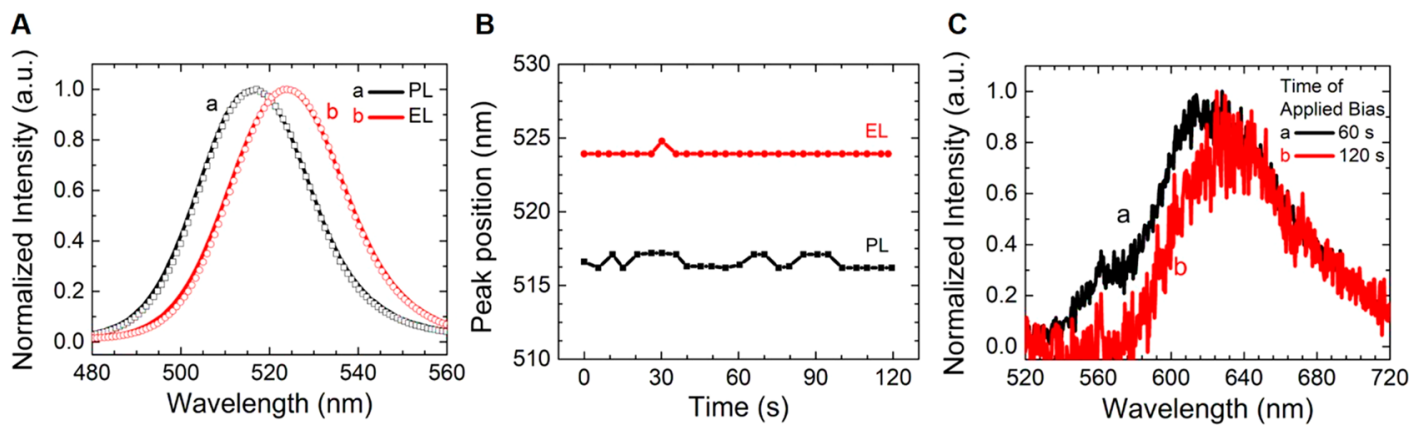

Figure 5. (A) Emission spectra as a function of time to observe the PL and EL features of $\mathrm{CsPbBr}_{3-x} \mathrm{I}_{x}$ nanoparticle-based LED devices with initial spectra (solid line) and spectra after $120 \mathrm{~s}$ emission (white dots). (B) Peak positions of both EL (red circles) and PL (black squares) as a function of time. (C) Emission spectra of halide-segregated CsPbBr ${ }_{3-x} \mathbf{I}_{x}$ bulk-based LED devices.

Scheme 1. Schematic Illustration of the Nanoparticle-to-Bulk Transformation in $\mathrm{CsPbBr}_{3-x} \mathrm{I}_{x}$ Perovskites under Annealing Process

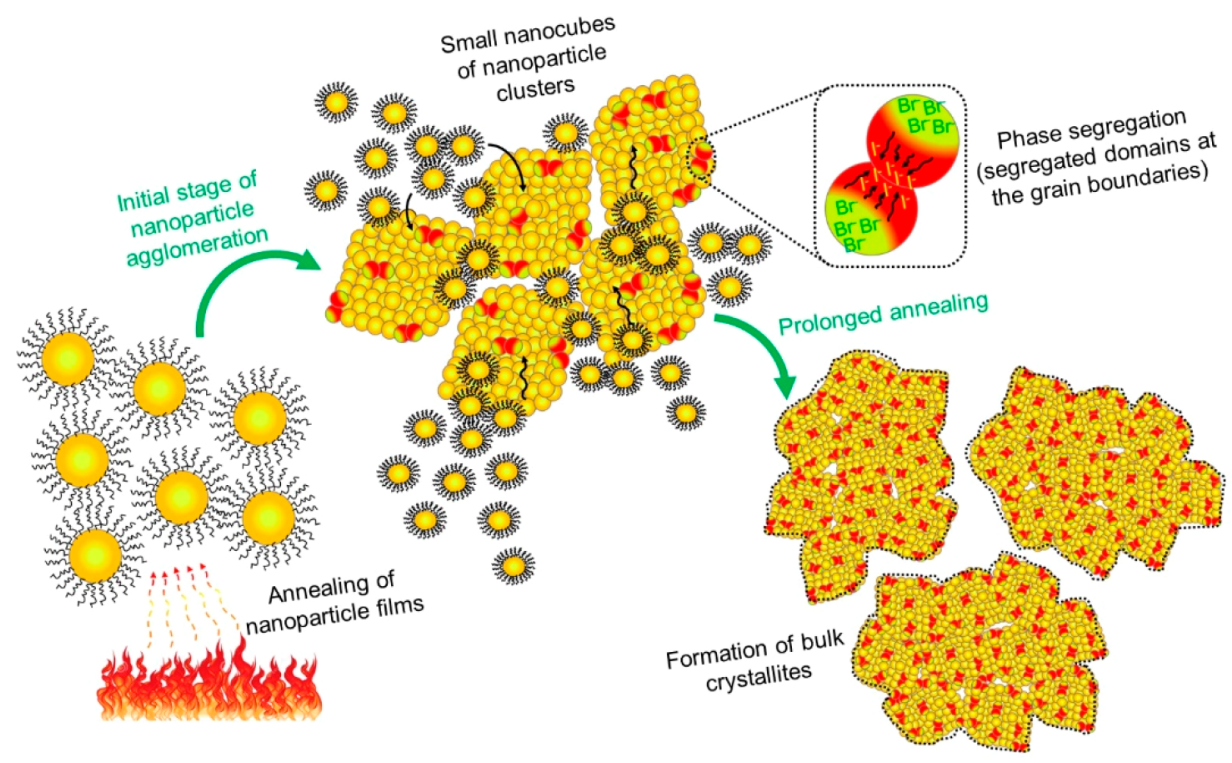

migration in single halide perovskites. ${ }^{43}$ In single halide perovskite, $\triangle \mathrm{CPP}$ varies and decreases from dark to light because of the ion vacancies' migration. However, an opposite trend is observed in mixed halide, where these vacancies are used as roads for the photoinduced migration ${ }^{42}$ of one of the halides, consequently producing the phase segregation and the observation of a different trend in the $\triangle \mathrm{CPP}$ by a different direction in the ion migration. Hence, it can be noted that the halide segregation is directly correlated with the ion migration, and it is dependent on the topography of the bulk film.

Light-Emitting Properties of Phase-Segregation-Free $C s \mathrm{PbBr}_{3-x} I_{x}$ Nanoparticle Films. After observing that $\mathrm{Cs} \mathrm{PbBr}_{3-x} \mathrm{I}_{x}$ NP films are free from halide segregation effects by tracking their photophysical properties, the materials were used in making LEDs. Here, the electroluminescence (EL) feature of the $\mathrm{CsPBr}_{3-x} \mathrm{I}_{x}$ nanoparticle film-based device $(x=$ 0.63 ) was studied (Figure 5). Application of an electrical field (9 V, Figure S21) induced a shift of EL peak with respect to the PL due to the Stark effect ${ }^{44}$ (see Figure 5A). Significantly, no shift of the EL peak position at $523 \mathrm{~nm}$ was observed at the working conditions of the device (Figure 5B), without any additional emission signals elsewhere. This fact indicates that perovskite NPs can produce segregation-free mixed halide devices with no shift of the emitted light wavelength. The latter can be also seen in Figure S20, where the green EL from the device was retained for $120 \mathrm{~s}$ without any color change. By contrast, we could observe the opposite trend at the annealed bulk film-based device (Figure 5C) where a progressive red shift in the emission wavelength was observed during the continuous work conditions under applied bias (9 V). The emission appeared at a wavelength of $623 \mathrm{~nm}$ after $60 \mathrm{~s}$. The initial emission peak position at $0 \mathrm{~s}$ was beyond the detection limit, deducing a low EL of the halide-segregated LED device. Furthermore, the emission peak position shifted to $633 \mathrm{~nm}$ after $120 \mathrm{~s}$ (see Figure 5C). Beyond the achieved red shift, the emission at $623 \mathrm{~nm}$ and continuous shift to $633 \mathrm{~nm}$ demonstrated the evolution of the I-rich segregated domain and enriching iodine composition in the segregated domain. The injected free carriers were trapped in the segregated I-rich domain, promoting radiative recombination as electroluminescence. Therefore, the two different electroluminescence values from nanoparticles to bulk film also demonstrated the segregation-free and stable emission from the nanoparticles.

Mechanism of Nanoparticle-to-Bulk Transformation under Annealing Process. According to the morphology, crystallinity, and photophysical features of the mixed halide perovskites described above, the relation between nanoparticle agglomeration and phase segregation is shown in Scheme 1. Under 
heating, in the early stage, the majority of ligands are burned out and $\mathrm{CsPbBr}_{3-x} \mathrm{I}_{x}$ NPs start to promote the growth of bigger grains with a nanocube-shaped morphology. The agglomerated large-sized grain nanocubes are produced, and the large nanocubes facilitate the halide segregation along GBs under photoirradiation. The agglomeration occurred with material migration from smaller NPs to larger NPs, where the formation of big particles is thermodynamically favored over the smaller ones. ${ }^{32}$ The phase segregation is intensified, as is evident in the generation of Br-rich and I-rich domains monitored by emission and the decrease of the green-color PL features in the annealed samples. In this context, mixed halide perovskite films with crystalline sizes less than $46 \pm 7 \mathrm{~nm}$ show high phase stability. Consequently, the preparation of phasesegregation-free mixed halide perovskites by acting on the maximum allowed grain size will help to improve the performance of optoelectronic devices built by using these perovskites.

In summary, to accurately determine the conditions in which a deleterious photoinduced phase segregation appears in mixed halide perovskite films, we have tracked the NP-to-bulk transition induced by annealing. No phase segregation was observed in nanoparticle films at any halide composition. According to the morphology, crystallinity, and photophysical properties of the $\mathrm{CsPbBr}_{3-x} \mathrm{I}_{x}$ perovskites, we found that halide segregation effect appears in nanocube grains formed during the agglomeration, with a threshold size around $46 \pm 7 \mathrm{~nm}$, in a process where organic capping ligands play a minor role. This measured grain size from SEM images has an outstanding agreement with the diffusion length calculated from eq 1 and the experimentally determined average lifetime $(45.8 \mathrm{~nm})$ for $\mathrm{PL}, K$, and $K_{\text {sat }}$ confirming the size threshold by two completely different sets of experimental measurements. The crystalline growth and nanoparticle capping removal generates grain boundaries, facilitating the formation and accumulation of segregated domains under photoirradiation; nevertheless, the process is counterbalanced with the confinement of carrier diffusion into the grains. Thus, for films constituted by grains with dimensions less than the diffusion length, a stabilized behavior is attained. A crystalline size lower than the later value allows the preparation of phase-segregation-free $\mathrm{CsPbBr}_{3-x} \mathrm{I}_{x}$ films by using nanoparticle solutions with different halide composition or variable synthesis conditions. This insight opens the possibility of preparing mixed halide perovskitebased materials with phase stability to improve the performances of optoelectronic devices.

\section{ASSOCIATED CONTENT}

\section{S Supporting Information}

The Supporting Information is available free of charge on the ACS Publications website at DOI: 10.1021/acsenergylett.8b02207.

Experimental section, UV-vis absorption, emission spectra, photo images of various $\mathrm{CsPbBr}_{3-x} \mathrm{I}_{x}$ films, morphological views of $\mathrm{CsPbBr}_{3-x} \mathrm{I}_{x}$ films through SEM measurement, XRD patterns, TEM images of $\mathrm{CsPbBr}_{3-x} \mathrm{I}_{x}$ QDs, and detailed diffusion length calculation (PDF)

\section{AUTHOR INFORMATION}

\section{Corresponding Author}

*E-mail: sero@uji.es. Twitter: @IvanMoraSero.

\section{ORCID}

Ángel M. Meléndez: 0000-0002-5166-1840

Martha E. Niño-Gómez: 0000-0002-2216-2348

Iván Mora-Seró: 0000-0003-2508-0994

\section{Author Contributions}

\#A.F.G.-R. and S.J.Y. contributed equally to this work.

\section{Notes}

The authors declare no competing financial interest.

\section{ACKNOWLEDGMENTS}

This work was supported by the University Jaume I (project SOLENPE UJI-B2016-05) and the European Research Council (ERC) via Consolidator Grant (724424 - NoLIMIT). In addition, we acknowledge the Spanish Ministry of Economy, Industry and Competitiveness (MINECO) and EU (FEDER) under project TEC2014-60173 and the Generalitat Valenciana under project Prometeo II 2015/004 and Prometeo/2018/098 (Q-Devices) for financial support. A.F.G.-R. acknowledges Colciencias for a Ph.D. 617-scholarship and Universidad Industrial de Santander through the Colciencias project 8836 , external code 110265843664 . The authors are also grateful to the SCSIE-University of Valencia for providing TEM microscopy facilities and SCSI-University Jaume I for its help with SEM and XRD characterization.

\section{REFERENCES}

(1) Research Cell Record Efficiency Chart. https://www.nrel.gov/ pv/assets/images/efficiency-chart.png.

(2) Brenner, T. M.; Egger, D. A.; Kronik, L.; Hodes, G.; Cahen, D. Hybrid Organic-inorganic Perovskites: Low-cost Semiconductors with Intriguing Charge-transport Properties. Nat. Rev. Mater. 2016, 1, 15007.

(3) Sutherland, B. R.; Sargent, E. H. Perovskite Photonic Sources. Nat. Photonics 2016, 10, 295-302.

(4) Tan, Z.-K.; Moghaddam, R. S.; Lai, M. L.; Docampo, P.; Higler, R.; Deschler, F.; Price, M.; Sadhanala, A.; Pazos, L. M.; Credgington, D.; et al. Bright Light-emitting Diodes Based on Organometal Halide Perovskite. Nat. Nanotechnol. 2014, 9, 687-692.

(5) Suárez, I.; Hassanabadi, E.; Maulu, A.; Carlino, N.; Maestri, C. A.; Latifi, M.; Bettotti, P.; Mora-Seró, I.; Martínez-Pastor, J. P. Integrated Optical Amplifier-Photodetector on a Wearable Nanocellulose Substrate. Adv. Opt. Mater. 2018, 6, 1800201.

(6) Suárez, I.; Juárez-Pérez, E. J.; Bisquert, J.; Mora-Seró, I.; Martínez-Pastor, J. P. Polymer/Perovskite Amplifying Waveguides for Active Hybrid Silicon Photonics. Adv. Mater. 2015, 27, 6157-6162.

(7) Xing, G.; Mathews, N.; Lim, S. S.; Yantara, N.; Liu, X.; Sabba, D.; Grätzel, M.; Mhaisalkar, S.; Sum, T. C. Low-temperature Solutionprocessed Wavelength-tunable Perovskites for Lasing. Nat. Mater. 2014, 13, 476-480.

(8) Zhu, H.; Fu, Y.; Meng, F.; Wu, X.; Gong, Z.; Ding, Q.; Gustafsson, M. V.; Trinh, M. T.; Jin, S.; Zhu, X. Y. Lead Halide Perovskite Nanowire Lasers with Low Lasing Thresholds and High Quality Factors. Nat. Mater. 2015, 14, 636-642.

(9) Rhee, J. H.; Chung, C.-C.; Diau, E. W.-G. A Perspective of Mesoscopic Solar Cells Based on Metal Chalcogenide Quantum Dots and Organometal-halide Perovskites. NPG Asia Mater. 2013, 5, No. e68.

(10) Noh, J. H.; Im, S. H.; Heo, J. H.; Mandal, T. N.; Seok, S. I. Chemical Management for Colorful, Efficient, and Stable InorganicOrganic Hybrid Nanostructured Solar Cells. Nano Lett. 2013, 13, 1764-1769.

(11) Suarez, B.; Gonzalez-Pedro, V.; Ripolles, T. S.; Sanchez, R. S.; Otero, L.; Mora-Sero, I. Recombination Study of Combined Halides (Cl, Br, I) Perovskite Solar Cells. J. Phys. Chem. Lett. 2014, 5, 16281635. 
(12) Hoke, E. T.; Slotcavage, D. J.; Dohner, E. R.; Bowring, A. R.; Karunadasa, H. I.; McGehee, M. D. Reversible Photo-induced Trap Formation in Mixed-halide Hybrid Perovskites for Photovoltaics. Chem. Sci. 2015, 6, 613-617.

(13) Li, W.; Rothmann, M. U.; Liu, A.; Wang, Z.; Zhang, Y.; Pascoe, A. R.; Lu, J.; Jiang, L.; Chen, Y.; Huang, F.; et al. Phase Segregation Enhanced Ion Movement in Efficient Inorganic CsPbIBr ${ }_{2}$ Solar Cells. Adv. Energy Mater. 2017, 7, 1700946.

(14) Yoon, S. J.; Kuno, M.; Kamat, P. V. Shift Happens. How Halide Ion Defects Influence Photoinduced Segregation in Mixed Halide Perovskites. ACS Energy Lett. 2017, 2, 1507-1514.

(15) Beal, R. E.; Slotcavage, D. J.; Leijtens, T.; Bowring, A. R.; Belisle, R. A.; Nguyen, W. H.; Burkhard, G. F.; Hoke, E. T.; McGehee, M. D. Cesium Lead Halide Perovskites with Improved Stability for Tandem Solar Cells. J. Phys. Chem. Lett. 2016, 7, 746-51.

(16) Yoon, S. J.; Draguta, S.; Manser, J. S.; Sharia, O.; Schneider, W. F.; Kuno, M.; Kamat, P. V. Tracking Iodide and Bromide Ion Segregation in Mixed Halide Lead Perovskites during Photoirradiation. ACS Energy Lett. 2016, 1, 290-296.

(17) Bischak, C. G.; Hetherington, C. L.; Wu, H.; Aloni, S.; Ogletree, D. F.; Limmer, D. T.; Ginsberg, N. S. Origin of Reversible Photoinduced Phase Separation in Hybrid Perovskites. Nano Lett. 2017, 17, 1028-1033.

(18) Samu, G. F.; Janáky, C.; Kamat, P. V. A Victim of Halide Ion Segregation. How Light Soaking Affects Solar Cell Performance of Mixed Halide Lead Perovskites. ACS Energy Lett. 2017, 2, 18601861

(19) Unger, E. L.; Kegelmann, L.; Suchan, K.; Sorell, D.; Korte, L.; Albrecht, S. Roadmap and Roadblocks for the Band gap Tunability of Metal Halide Perovskites. J. Mater. Chem. A 2017, 5, 11401-11409.

(20) Bailie, C. D.; McGehee, M. D. High-efficiency Tandem Perovskite Solar Cells. MRS Bull. 2015, 40, 681-686.

(21) Yang, T. C.-J.; Fiala, P.; Jeangros, Q.; Ballif, C. High-Bandgap Perovskite Materials for Multijunction Solar Cells. Joule 2018, 2, $1421-1436$.

(22) Gualdrón-Reyes, A. F.; Yoon, S. J.; Mora-Seró, I. Recent Insights for Achieving Mixed Halide Perovskites without Halide Segregation. Curr. Opin. Electrochem. 2018, In press, DOI: 10.1016/ j.coelec.2018.09.007.

(23) Yi, C.; Luo, J.; Meloni, S.; Boziki, A.; Ashari-Astani, N.; Gratzel, C.; Zakeeruddin, S. M.; Rothlisberger, U.; Gratzel, M. Entropic Stabilization of Mixed A-cation $\mathrm{ABX}_{3}$ Metal Halide Perovskites for High Performance Perovskite Solar Cells. Energy Environ. Sci. 2016, 9, 656-662.

(24) Braly, I. L.; Stoddard, R. J.; Rajagopal, A.; Uhl, A. R.; Katahara, J. K.; Jen, A. K. Y.; Hillhouse, H. W. Current-Induced Phase Segregation in Mixed Halide Hybrid Perovskites and its Impact on Two-Terminal Tandem Solar Cell Design. ACS Energy Lett. 2017, 2, $1841-1847$.

(25) Slotcavage, D. J.; Karunadasa, H. I.; McGehee, M. D. LightInduced Phase Segregation in Halide-Perovskite Absorbers. ACS Energy Lett. 2016, 1, 1199-1205.

(26) Yang, Z.; Rajagopal, A.; Jo, S. B.; Chueh, C. C.; Williams, S.; Huang, C. C.; Katahara, J. K.; Hillhouse, H. W.; Jen, A. K. Stabilized Wide Bandgap Perovskite Solar Cells by Tin Substitution. Nano Lett. 2016, 16, 7739-7747.

(27) Hu, M.; Bi, C.; Yuan, Y.; Bai, Y.; Huang, J. Stabilized Wide Bandgap $\mathrm{MAPbBr}_{\mathrm{x}} \mathrm{I}_{3-\mathrm{x}}$ Perovskite by Enhanced Grain Size and Improved Crystallinity. Adv. Sci. 2016, 3, 1500301.

(28) Abdi-Jalebi, M.; Andaji-Garmaroudi, Z.; Cacovich, S.; Stavrakas, C.; Philippe, B.; Richter, J. M.; Alsari, M.; Booker, E. P.; Hutter, E. M.; Pearson, A. J.; et al. Maximizing and Stabilizing Luminescence from Halide Perovskites with Potassium Passivation. Nature 2018, 555, 497-501.

(29) Draguta, S.; Sharia, O.; Yoon, S. J.; Brennan, M. C.; Morozov, Y. V.; Manser, J. S.; Kamat, P. V.; Schneider, W. F.; Kuno, M. Rationalizing the Light-induced Phase Separation of Mixed Halide Organic-inorganic Perovskites. Nat. Commun. 2017, 8, 200.
(30) Protesescu, L.; Yakunin, S.; Bodnarchuk, M. I.; Krieg, F.; Caputo, R.; Hendon, C. H.; Yang, R. X.; Walsh, A.; Kovalenko, M. V. Nanocrystals of Cesium Lead Halide Perovskites $\left(\mathrm{CsPbX}_{3}, \mathrm{X}=\mathrm{Cl}, \mathrm{Br}\right.$, and I): Novel Optoelectronic Materials Showing Bright Emission with Wide Color Gamut. Nano Lett. 2015, 15, 3692-3696.

(31) Nedelcu, G.; Protesescu, L.; Yakunin, S.; Bodnarchuk, M. I.; Grotevent, M. J.; Kovalenko, M. V. Fast Anion-Exchange in Highly Luminescent Nanocrystals of Cesium Lead Halide Perovskites (CsPbX $3, \mathrm{X}=\mathrm{Cl}, \mathrm{Br}, \mathrm{I})$. Nano Lett. 2015, 15, 5635-40.

(32) Hoffman, J. B.; Zaiats, G.; Wappes, I.; Kamat, P. V. CsPbBr Solar Cells: Controlled Film Growth through Layer-by-Layer Quantum Dot Deposition. Chem. Mater. 2017, 29, 9767-9774.

(33) Brennan, M. C.; Draguta, S.; Kamat, P. V.; Kuno, M. LightInduced Anion Phase Segregation in Mixed Halide Perovskites. ACS Energy Lett. 2018, 3, 204-213.

(34) Tang, X.; van den Berg, M.; Gu, E.; Horneber, A.; Matt, G. J.; Osvet, A.; Meixner, A. J.; Zhang, D.; Brabec, C. J. Local Observation of Phase Segregation in Mixed-Halide Perovskite. Nano Lett. 2018, 18, 2172-2178.

(35) Zhou, L.; Yu, K.; Yang, F.; Cong, H.; Wang, N.; Zheng, J.; Zuo, Y.; Li, C.; Cheng, B.; Wang, Q. Insight into the Effect of Ligandexchange on Colloidal CsPbBr 3 Perovskite Quantum dot/mesoporous- $\mathrm{TiO}_{2}$ Composite-based Photodetectors: Much Faster Electron Injection. J. Mater. Chem. C 2017, 5, 6224-6233.

(36) Draguta, S.; Thakur, S.; Morozov, Y. V.; Wang, Y.; Manser, J. S.; Kamat, P. V.; Kuno, M. Spatially Non-uniform Trap State Densities in Solution-Processed Hybrid Perovskite Thin Films. J. Phys. Chem. Lett. 2016, 7, 715-721.

(37) Sutton, R. J.; Eperon, G. E.; Miranda, L.; Parrott, E. S.; Kamino, B. A.; Patel, J. B.; Hörantner, M. T.; Johnston, M. B.; Haghighirad, A. A.; Moore, D. T.; Snaith, H. J. Bandgap-Tunable Cesium Lead Halide Perovskites with High Thermal Stability for Efficient Solar Cells. Adv. Energy Mater. 2016, 6, 1502458.

(38) Swarnkar, A.; Marshall, A. R.; Sanehira, E. M.; Chernomordik, B. D.; Moore, D. T.; Christians, J. A.; Chakrabarti, T.; Luther, J. M. Quantum Dot-induced Phase Stabilization of Alpha-CsPbI ${ }_{3}$ Perovskite for High-efficiency Photovoltaics. Science 2016, 354, 92-95.

(39) Vitoreti, A. B. F.; Agouram, S.; Solis de la Fuente, M.; MuñozSanjosé, V.; Schiavon, M. A.; Mora-Seró, I. Study of the Partial Substitution of $\mathrm{Pb}$ by $\mathrm{Sn}$ in $\mathrm{Cs}-\mathrm{Pb}-\mathrm{Sn}-\mathrm{Br}$ Nanocrystals Owing to Obtaining Stable Nanoparticles with Excellent Optical Properties. J. Phys. Chem. C 2018, 122, 14222-14231.

(40) Bekenstein, Y.; Koscher, B. A.; Eaton, S. W.; Yang, P.; Alivisatos, A. P. Highly Luminescent Colloidal Nanoplates of Perovskite Cesium Lead Halide and Their Oriented Assemblies. J. Am. Chem. Soc. 2015, 137, 16008-16011.

(41) Yuan, Y. B.; Huang, J. S. Ion Migration in Organometal Trihalide Perovskite and Its Impact on Photovoltaic Efficiency and Stability. Acc. Chem. Res. 2016, 49, 286-293.

(42) Ruth, A.; Brennan, M. C.; Draguta, S.; Morozov, Y. V.; Zhukovskyi, M.; Janko, B.; Zapol, P.; Kuno, M. Vacancy-Mediated Anion Photosegregation Kinetics in Mixed Halide Hybrid Perovskites: Coupled Kinetic Monte Carlo and Optical Measurements. ACS Energy Lett. 2018, 3, 2321-2328.

(43) Azpiroz, J. M.; Mosconi, E.; Bisquert, J.; De Angelis, F. Defect Migration in Methylammonium Lead Iodide and Its Role in Perovskite Solar Cell Operation. Energy Environ. Sci. 2015, 8, 2118-2127.

(44) Clasen Hames, B.; Sánchez Sánchez, R. 1.; Fakharuddin, A.; Mora-Seró, I. A Comparative Study of Light-Emitting Diodes Based on All-Inorganic Perovskite Nanoparticles $\left(\mathrm{CsPbBr}_{3}\right)$ Synthesized at Room Temperature and by a Hot-Injection Method. ChemPlusChem 2018, 83, 294-299. 